\title{
Chinese expert consensus on the standardized procedure and technique of transbronchial cryobiopsy
}

Shuliang Guo ${ }^{1}$, Qiang Li $^{2}$, Jinyue Jiang ${ }^{1}$, Fengming Luo ${ }^{3}$, Yishi Li $^{1}$, Faguang Jin ${ }^{4}$, Xinzhu Liu ${ }^{1}$, Hongwu Wang ${ }^{5}$, Ping Chen ${ }^{1}$, Chong Bai ${ }^{6}$, Haiyun Dai ${ }^{1}$, Haidong Huang ${ }^{6}$, Xianwei Ye ${ }^{7}$, Xianghua Yi $^{8}$, Jie Zhang ${ }^{9}$, Changhui Wang ${ }^{10}$, Mingyao Ke ${ }^{11}$, Jiayuan Sun ${ }^{12}$, Jing Feng ${ }^{13}$, Hongmei Zhou ${ }^{14}$, Youru $\mathrm{Wu}^{15}$, Zhen Wang ${ }^{16}$, Yun $\mathrm{Ma}^{17}$, Jing $\mathrm{Li}^{18}$, Liping $\mathrm{Lv}^{19}$, Baosong Xie ${ }^{20}$, Wolfgang Hohenforst-Schmidt ${ }^{21}$, Weimin Ding ${ }^{22}$, Xiaoping Wang ${ }^{23}$, Junyong Yang ${ }^{24}$, Qingshan Cai ${ }^{25}$, Peng Sun ${ }^{26}$, Zhuang Luo ${ }^{27}$, Mohan Giri $^{1}$; on behalf of the Working Committee on Interventional Pulmonology, Respiratory Physicians' Branch of Chinese Medical Doctor Association, Committee of Interventional Pulmonology, Chinese Society for Tuberculosis, Chinese Medical Association, Chinese Western Association of Interventional Pulmonology

${ }^{1}$ The First Affiliated Hospital of Chongqing Medical University, Chongqing 400016, China; ${ }^{2}$ Shanghai East Hospital, Tong Ji University, Shanghai 200120, China; ${ }^{3}$ West China Hospital, Sichuan University, Chengdu 610041, China; ${ }^{4}$ Tangdu Hospital Affiliated to Air Force Medical University, Xi' ${ }^{2}$ 710038, China; ${ }^{5}$ Emergency General Hospital, Beijing 100028, China; ${ }^{6}$ Changhai Hospital, The Second Military Medical University, Shanghai 200433 , China; ${ }^{7}$ Guizhou Provincial People's Hospital, Guiyang 550002, China; ${ }^{8}$ Tongji Hospital of Tongji University, Shanghai 200065, China; ${ }^{9}$ Beijing Tiantan Hospital, Capital Medical University, Beijing 100070, China; ${ }^{10}$ Shanghai Tenth People's Hospital, Tongji University, Shanghai 200072, China;

${ }^{11}$ The Second Affiliated Hospital of Xiamen Medical College, Xiamen 361021, China; ${ }^{12}$ Shanghai Chest Hospital, Shanghai Jiao Tong University, Shanghai 200030, China; ${ }^{13}$ General Hospital, Tianjin Medical University, Tianjin 300052, China; ${ }^{14}$ Zhongshan Hospital Affiliated to Guangdong Medical University, Zhongshan 528415, China; ${ }^{15}$ Mianyang Central Hospital, Mianyang 621000, China; ${ }^{16}$ Beijing Chao-yang Hospital, Capital Medical University, Beijing 100069, China; ${ }^{17}$ Henan Provincial People’s Hospital, Zhengzhou 450003, China; ${ }^{18}$ Guangdong Provincial People's Hospital, Guangzhou 510030, China; ${ }^{19}$ Anhui Chest Hospital, Hefei 230022, China; ${ }^{20}$ Fujian Provincial Hospital, Fuzhou 350001, China; ${ }^{21}$ "Hof” Clinics, University of Erlangen, Hof, Germany; ${ }^{22}$ Beijing Chest Hospital, Capital Medical University, Beijing 101149 , China; ${ }^{23}$ Shandong Provincial Chest Hospital, Jinan 250000, China; ${ }^{24}$ Xinjiang Chest Hospital, Urumqi 830000, China; ${ }^{25}$ Hangzhou Red Cross Hospital, Hangzhou 310003, China; ${ }^{26} \mathrm{Jilin}$ Tuberculosis Hospital, Changchun 212006, China; ${ }^{27}$ First Affiliated Hospital of Kunming Medical University, Kunming 650031, China

Correspondence to: Shuliang Guo. Department of Respiratory and Critical Care Medicine, The First Affiliated Hospital of Chongqing Medical University, Chongqing 400016, China. Email: guos1999@sina.com.

Submitted Sep 18, 2019. Accepted for publication Nov 29, 2019.

doi: $10.21037 /$ jtd.2019.12.36

View this article at: http://dx.doi.org/10.21037/jtd.2019.12.36

\section{Introduction}

Transbronchial cryobiopsy (TBCB) is a technique to obtain frozen samples of lung tissue from the distal bronchioles, in which the tissue surrounding the tip of a cryoprobe inserted through the bronchoscope is frozen with a rapid freezing process and abruptly pulled away from adherent tissue. The advantages of TBCB are improved diagnostic yield, reduced trauma, large and high-quality specimens, potentially decreased complications, and lower cost in comparison to surgical lung biopsy (SLB). TBCB is mainly used for the etiological diagnosis of diffuse parenchymal lung diseases (DPLD) $(1,2)$. Additionally, it can be used for biopsy of peripheral lung lesions. TВCB has been widely used as a new diagnostic tool in Europe since 2009 (1), and has been used in some highly specialized interventional centers in China since 2015 (3). However, the procedure of ТВCВ has not been standardized. There are still disagreements among pulmonologists internationally and in China regarding operation methods, biopsy sites, number of biopsy specimens, types of frozen probes, freezing time and specimen handling, and there is urgent need for procedural standardization of TBCB for effectiveness and safety. This work is an evidence- and expert-based consensus on the procedure and technique of TBCB, which is a collaborative 
effort of the Working Committee on Interventional Pulmonology, Respiratory Physicians' Branch of Chinese Medical Doctor Association, Committee of Interventional Pulmonology, Chinese Society for Tuberculosis, Chinese Medical Association, and the Chinese Western Association of Interventional Pulmonology. We hope it will contribute to the transition of the etiologic diagnosis of DPLD from the clinico-radiologic (CR) model to the clinico-radiologicpathologic (CRP) model, which hopefully will increase the rate of etiologic diagnosis of DPLD, and improves the effectiveness, safety and standardization of the procedure and technique of TBCB to ensure diagnostic yield.

\section{Indications and contraindications}

\section{Indications}

\section{DPLD}

The main indication for TBCB is DPLD, which is a group of diffuse lung diseases that mainly affect the pulmonary interstitial and alveolar spaces, resulting in the loss of alveolar-capillary functional units characterized by extensive lesions in radiology. The etiology of DPLD is complex and difficult to diagnose. SLB has been regarded as the golden standard in the diagnosis of DPLD, but its clinical application is limited by severe surgical trauma, high cost and cardiopulmonary function requirements. On the other hand, samples obtained by the traditional transbronchial forceps biopsy (TBFB) and percutaneous lung biopsy (PCLB) are often inadequate in size and quality to fully represent the heterogeneity of the lesions and therefore insufficient for pathological diagnosis $(1,2,4)$. TBCB has been performed in thousands of DPLD patients worldwide, and a large number of studies have demonstrated a high diagnostic yield of over $80 \%$ in DPLD and low incidence of serious complications, and therefore it is considered a promising alternative to SLB in the diagnosis of DPLD $(3,5,6)$. When high resolution computed tomography (HRCT) findings combined with clinical and laboratory data (such as sputum examination, blood examination, routine bronchoscopy, etc.) is insufficient to make a diagnosis of DPLD, it is recommended to perform TBCB for a definite diagnosis, and SLB should be performed in the few patients whose etiology is still unclear after TBCB.

\section{Peripheral pulmonary lesion}

The etiological diagnosis of peripheral pulmonary lesions is another challenge for clinicians. With the development of ultra-fine bronchoscopy, ultrasound bronchoscopy, navigation bronchoscopy and other technologies in diagnostic bronchoscopy in recent years, the etiological diagnosis rate of peripheral pulmonary nodules has been significantly improved. Studies have shown that for the etiological diagnosis of peripheral solid pulmonary nodules such as lung cancer, the diagnostic yield of TBCB is similar or slightly higher than $\operatorname{TBFB}(5,7)$, which may be attributed to the homogeneity of solid pulmonary nodules such as tumors and hence the ease of diagnosis with cytological or small sample histological examination as in the case of TBFB. Therefore, for peripheral pulmonary lesions, it is recommended that TBFB be performed with the guidance of a radial ultrasound probe and/or C-arm X-ray before TBCB which should only be considered when the result of TBFB is negative. However, as TBCB can obtain larger and higher quality tissue specimens than TBFB (7), it is beneficial for further molecular pathological examination.

Further studies are necessary in order to conclude the value of TBCB in the etiological diagnosis of peripheral pulmonary lesions. At present TBCB is not recommended as the first choice for the diagnosis of peripheral pulmonary lesions.

\section{Surveillance of rejection after lung transplantation}

Patients with lung transplantation are prone to bronchiolitis obliterans syndrome (BOS), and the incidence of BOS is positively correlated with its mortality (8). Acute cell rejection and lymphocytic bronchiolitis are the main risk factors for the development of BOS. There is no effective treatment for BOS, so timely monitoring, early detection and early treatment of acute cell rejection are important to reduce the mortality among lung transplantation patients. Previously, the primary method for assessing rejection after lung transplantation was the use of standard biopsy forceps for TBFB (9), but current studies have shown that TBCB provides larger and higher quality tissue samples and is a safe alternative to conventional TBFB to monitor rejection after lung transplantation $(8,9)$.

\section{Contraindications}

\section{Absolute contraindication}

(I) Contraindications to the routine bronchoscopy procedure.

(II) Uncorrected coagulopathy, unstable hemodynamics, and severe respiratory failure $\left(\mathrm{PaO}_{2}\right.$ below 
$60 \mathrm{mmHg}$ even after supplemental oxygen or mechanical ventilation).

(III) Idiopathic pulmonary fibrosis (IPF) and other DPLD which could be clearly diagnosed with HRCT.

\section{Relative contraindications (10)}

(I) Uncontrolled hypertension or pulmonary arterial hypertension.

(II) Pulmonary dysfunction: diffusing capacity of the lungs for carbon monoxide (DLCO) $<35 \%$ or forced vital capacity (FVC) $<50 \%$.

(III) Adjacency of the localized lesion for biopsy to medium and large blood vessels, cavity or bullae of the lung.

(IV) Contraindications for rigid bronchoscopy or tracheal intubation. It has been reported that in these patients TBCB can be carried out under the laryngeal mask (11), but there are major safety concerns including poor control of bleeding, frozen vocal cord, vocal cord injury and asphyxia, etc.

\section{The operation procedure, methods and key technical points}

\section{Preoperative preparation}

\section{Preoperative examination and communication}

Chest high resolution CT and lung function test should be performed before the procedure. Patients with suspected pulmonary arterial hypertension should be evaluated with transthoracic echocardiography. The process, necessity, effectiveness, safety issues, cost-effectiveness, benefits and risks of the procedure and possible alternatives should be fully discussed with patients and families. Other preparations are the same as that for conventional bronchoscopy.

\section{Apparatuses and drugs}

Cryotherapy instrument, sterilized (low temperature plasma, etc.) cryoprobe, rigid bronchoscope or endotracheal intubation equipment, hemostasis occlusion balloon (stone extraction balloon or dilatation balloon), hemostatic drugs, closed thoracic drainage equipment, specimen collection and preservation equipment (including specimen bottles with $10 \%$ neutral buffered formalin or $2.5 \%$ glutaraldehyde fixative, pathogenic microbial culture flasks and tools for sample measurement, etc.). Other instruments are the same as that for conventional bronchoscopy. It is recommended that TBCB be carried out under the guidance of C-arm, radial ultrasonic probe and other visual equipment if available, so as to facilitate accurate positioning and reduce the occurrence of complications such as pneumothorax and hemorrhage $(10,12,13)$, while electromagnetic navigation equipment is more effective for the rapid positioning of peripheral lung lesions.

\section{Preoperative multidisciplinary discussion}

Preoperative multidisciplinary committee discussion involving expert clinicians, radiologists, anesthesiologists and pathologists is recommended to jointly formulate biopsy plans and risk mitigation and contingency plans, to ensure the quality and safety of the biopsy procedure.

\section{Procedure}

\section{Anesthesia}

It is recommended to perform TBCB under general anesthesia, while intravenous anesthesia is another option given the proficiency of the bronchoscopists, but it is not recommended to perform it under local anesthesia.

\section{Establishment of artificial airway}

It is strongly recommended to use a rigid bronchoscope or endotracheal tube (ETT) as the working channel in order to permit passage for the flexible bronchoscope and the cryoprobe, protect the vocal cords and control bleeding. Rigid bronchoscope is preferred if conditions permit. There have been reports of TBCB using a laryngeal mask (14), but it may significantly increase the risk of bleeding and frozen vocal cords (which may lead to asphyxia and vocal cord injury) and therefore is not recommended from a safety point of view.

\section{(I) Intubation of the rigid bronchoscope}

It is the same as routine rigid bronchoscopy intubation. The tip wall of the barrel should be placed tightly against the tracheal wall to avoid accidental damage to the flexible bronchoscope. The head of the rigid bronchoscope is attached to a high frequency jet ventilator or anesthetic machine, and the ventilation parameters and precautions for routine rigid bronchoscopy still apply in this procedure.

\section{(II) Endotracheal intubation}

It is the same as routine endotracheal intubation, and could be performed under general anesthesia or deep intravenous anesthesia. A 7.5 to $8.5 \mathrm{~mm}$ internal diameter ETT should 
be used to permit passage for the flexible bronchoscope and balloon catheters. The ventilation parameters and precautions for routine bronchoscopy with ETT apply in this procedure.

\section{Balloon catheters: inserted or standby}

When TBCB is performed with rigid bronchoscopy, it is not necessary to insert a balloon catheter before the biopsy, but the balloon catheter needs to be prepared on standby. When TBCB is performed with ETT, a balloon catheter inserted in advance is recommended in order to prevent massive hemorrhage. The balloon catheter can be inserted as follows. The flexible bronchoscope is inserted nasally through the glottis into the trachea alongside the ETT, the ETT balloon is then deflated, and the bronchoscope is advanced into the target bronchus of biopsy. A guide wire is inserted through the working channel of the bronchoscope into the target bronchus, the bronchoscope is then withdrawn and reinserted through the ETT into the trachea, and the balloon catheter is inserted along the guide wire into the target bronchus. The balloon is inflated under visual inspection to determine the amount of injected air needed for proper blockage of the target bronchus, and is then deflated and ready for use (15). The ETT balloon is then inflated again and the balloon catheter is stabilized with tapes at the nose and assistants if necessary.

\section{Cryobiopsy}

\section{(I) Site of biopsy}

With diffuse lesions, samples should be taken 1-2 cm from the visceral pleura most dense with exudative lesions, at different segments in the same lobe or separate ipsilateral lobes for a higher diagnostic yield (16). Samples should also be taken at normal-lesion borderlines and radiologically normal sites as comparison. Of note, the following sites should be avoided: fibrotic lesions (limited diagnostic value and increased pneumothorax risk), lesions located within $1 \mathrm{~cm}$ from the visceral pleura [increased pneumothorax risk (17)], central airway (unable to obtain bronchiole and alveolar tissue), conducting bronchi (accompanying vessels increase risk of massive hemorrhage), cavities (potential aneurysms may lead to massive hemorrhage) and swollen bronchi with chronic inflammation and contact bleeding. Biopsy of both lungs should not be performed in one procedure, or once hemorrhage or pneumothorax occurs it can be fatal.

\section{(II) Insertion of the flexible bronchoscope}

The flexible bronchoscope is inserted through the rigid barrel or the ETT and advanced to the entrance of the target bronchus. Localized lesions may require visualization equipment such as radial ultrasound, C-arm X-ray or navigation as guidance to ascertain the target bronchus.

\section{(III) Cryoprobe options}

Cryoprobes of either 2.4 or $1.9 \mathrm{~mm}$ diameters could be utilized in TBCB (1,17-19), but the former is more efficient and therefore a longer freezing time may be necessary for the latter to acquire the same area of samples $(2,10)$. However, the $1.9 \mathrm{~mm}$ cryoprobe is preferred when the target bronchus is small or situated distal in the upper lobe. Use of a $1.1 \mathrm{~mm}$ diameter cryoprobe has been reported in animal experiments $(20,21)$, but its diagnostic value in clinical settings is yet to be confirmed. Before the procedure, the cryoprobe should be connected to the cryosurgical unit and tested for its freezing efficacy in a water bath.

\section{(IV) Insert the cryoprobe to the target site}

Biopsy of diffuse lesions does not need radiological guidance, while $\mathrm{C}$-arm $\mathrm{X}$-ray is useful to ascertain the distance of the tip from the pleura and to locate the lesions, thus reducing risk of pneumothorax. Localized lesions require visualization equipment such as radial ultrasound, $\mathrm{C}$-arm X-ray or navigation to locate the target bronchus. $\mathrm{C}$-arm X-ray provides real-time guidance for precise location of the biopsy, which is recommended for the procedure.

When the flexible bronchoscope reaches the entrance of the target bronchus, the cryoprobe is inserted through the working channel into the target bronchus. If a balloon catheter is in place, the probe enters the target bronchus alongside the balloon catheter. When sampling for diffuse lesions without radiological guidance, the cryoprobe is inserted until it could no longer advance further, which indicates that the probe has reached the visceral pleura, and then withdrawn 1-2 cm to perform TBCB. The insertionwithdrawn process could be repeated several times for confirmation. The insertion of the probe should be gentle, especially when using the $1.9 \mathrm{~mm}$ probe, or the pleura may be punctured causing pneumothorax. If the target bronchus is too small to contain the probe tip, it could be dilated with a small amount of air or saline before sampling, or a change in biopsy site or probe diameter could be made. When sampling for a localized lesion, in case of a swollen bronchus, administer $1 \mathrm{~mL}$ of cold adrenaline $(1: 10,000)$ and $2 \mathrm{~mL}$ of budesonide solution to the target bronchus, and proceed after the swelling eliminates. For sampling of diffuse lesions, if the 
target bronchi are swollen, cryobiopsy should be performed in an alternative target site which is not swollen.

\section{(V) Cryobiopsy}

Once the cryoprobe reaches the target site, set the $\mathrm{CO}_{2}$ pressure to 50-60 bar, pedal for several seconds and then withdraw the cryoprobe together with the flexible bronchoscope, and collect the tissue at the probe tip for pathological evaluation. Freezing time of $3-6$ and $6-7 \mathrm{~s}$ is recommended for the 2.4 and $1.9 \mathrm{~mm}$ probe, respectively (1-4,6,7,10-14,16-19). Excessively violent pulling of the probe should be avoided. If the cryoprobe cannot be withdrawn, the tissue should be thawed and then apply a more appropriate freezing time.

The harvested tissue area is associated with multiple factors including freezing time, diameter of the cryoprobe, condition of the cryoprobe, gas pressure and moisture content and tenacity of the tissue. Higher gas pressure requires shorter freezing time. For efficacy and safety concerns, a trial of 3 and $5 \mathrm{~s}$ for 2.4 and $1.9 \mathrm{~mm}$ probes is recommended, respectively, and the freezing time could be adjusted according to the harvested sample size until a satisfactory size of biopsy is obtained $(10,22)$. As suggested by pathologists, samples with $\geq 5 \mathrm{~mm}$ diameter provide sufficient tissue for evaluation in most cases (23).

\section{(VI) Preservation of samples}

See section "Preservation, handling and examination of the specimens".

\section{(VII) Observation for hemorrhage and pneumothorax}

When TBCB is performed with ETT, a balloon is inflated with the tested amount of air immediately after withdrawal of the cryoprobe. Once the sample is removed, the flexible bronchoscope is reinserted to examine the balloon and bleeding. If there is no blood seeping out of the sampled site, the balloon is carefully deflated, and inflated immediately if any signs of bleeding occur. Meanwhile, the patient is closely examined for pneumothorax and subcutaneous emphysema. If the procedure is carried out with $\mathrm{C}$-arm X-ray, pneumothorax could be observed visually. When TBCB if performed with rigid bronchoscopy, the flexible bronchoscope is reinserted to examine the bleeding once the sample is removed. See part "Management of common complications" for management of hemorrhage and pneumothorax. Another sample could be taken if there is no pneumothorax or hemorrhage, or if existing hemorrhage has been controlled.

(VIII) Repeated cryobiopsy in one procedure

The cryoprobe enters the biopsy site again for repeated biopsy. When TBCB is performed with ETT and repeated biopsy is needed in another lobe, the balloon catheter should be redirected to the bronchus of the target segment before the biopsy, which could be achieved with the following technique: withdraw the balloon catheter into the lobar or main bronchus, insert the guide wire through the catheter and redirect the catheter into the next target bronchus with forceps under flexible bronchoscope. In the case of TBCB with rigid bronchoscopy, a repeated cryobiopsy could be made immediately after the previous one as the balloon catheter is on standby.

Times of biopsy and number of specimens are associated with the area of the specimens, category of diseases and intended examinations of the specimen as well as risk of complications, and consensus has not been reached on the optimum number of harvested samples. In most interventional centers in China, 3-5 specimens are obtained for examinations of pathology and pathogens. Rapid on-site evaluation (ROSE) is highly recommended if it is available, which provides instant assessment on quality of specimens and preliminary identification of tumor, tuberculosis and fungus, etc. and therefore minimizes the number of samples needed, reduces duration of the procedure and risk of pneumothorax, hemorrhage and anesthetic accidents $(24,25)$. The value of ROSE for evaluation and diagnosis of interstitial lung diseases has yet to be determined.

\section{(IX) Conclusions of the procedure}

When the desired specimens have been obtained, if no active hemorrhage is present to the flexible bronchoscopy examination and vital signs are stable, the rigid bronchoscope could be removed and then the patient should be escorted to post-anesthesia care unit or intensive care unit with ventilation through endotracheal intubation or laryngeal mask.

\section{Post-operative observation and management}

The patient should be closely observed for signs of pneumothorax, mediastinal emphysema or subcutaneous emphysema and chest $\mathrm{X}$-ray should be completed within 2 hours post-operatively (26), while delayed pneumothorax has also been reported to happen within 72 hours after the procedure, which should be managed promptly.

Hemostatics and expectorants could be given as needed to prevent hemorrhage and obstruction of small airways with secretion and blood clot. Short-term antibiotics could be administered for patients highly prone to infection, such as immunocompromised patients. 
Preservation, handling and examination of the specimens The harvested frozen specimen is placed in $37^{\circ} \mathrm{C}$ or room temperature saline with the cryoprobe to thaw and then removed from the probe with a wet gauze. Violent removal of the specimen should be avoided. The specimen removed should be measured for its length, width and thickness within $1 \mathrm{~min}$ and then immediately immersed into $10 \%$ neutral buffered formalin (for pathological examination) or saline (for pathogen culture). In addition, the specimen should be cut into blocks of $1 \mathrm{~mm}^{3}$ and preserved in $2.5 \%$ glutaraldehyde fixative at $4{ }^{\circ} \mathrm{C}$ for 2 hours if electron microscopic examination is indicated. Formalin-fixed specimens should be delivered to pathologic evaluation within 6 hours and made into paraffin section slides which go through routine optical microscopic examination with hematoxylin and eosin stain and optionally special stains and immunohistochemistry as decided by pathologists. After initial examinations, the remainder of the specimens should be preserved in paraffin in case of further examinations needed, such as special stains and molecular pathology examinations. Specimens in saline should be delivered to laboratory for microbial culture $(10,15)$.

\section{Diagnosis}

Despite the large size of samples obtained with TBCB which is favorable to a pathologic or pathogenic diagnosis, collaboration of pathologist with physicians and radiologist (i.e., the CRP precise diagnostic model) is preferred for diagnosis of interstitial lung diseases.

\section{Summary of key points in the procedure of TBCB}

* It should be conducted with general anesthesia or deep intravenous anesthesia;

* Artificial airway with rigid bronchoscopy or endotracheal intubation is recommended;

* Biopsy should be performed in extensive lesions for diffuse lesions or with the visualized guidance of radial ultrasound, C-arm X-ray or electromagnetic navigation equipment;

* Recommended freezing time is 3-6 s for the $2.4 \mathrm{~mm}$ probe and 6-7 $\mathrm{s}$ for the $1.9 \mathrm{~mm}$ probe;

* Specimens should be harvested with $\geq 5 \mathrm{~mm}$ diameter, and number of specimens obtained should be 3-5; and

* Thawed specimens should be handled and delivered for examination as soon as possible, and diagnoses should be reached with the CRP model.

\section{Management of common complications}

Complications directly associated with TBCB include hemorrhage, pneumothorax, mediastinal and subcutaneous emphysema, post-operative infection and acute exacerbation of the disease. Indirect complications include those associated with bronchoscopy, anesthesia and mechanical ventilation. In general, complication rates of TBCB are significantly lower than that of SLB (27). The rate of pneumothorax in TBCB is similar to that in TBFB, while divergent opinions exist as for the rate of hemorrhage in TBCB and TBLB with some report no significant difference $(5,28)$ and others claim significantly higher incidence of mild to moderate hemorrhage in TBCB $(6,12,29)$. The risk of hemorrhage does require special attention and instant management from a safety point of view. Major complications directly associated with ТВСВ and relevant management is discussed herein.

\section{Hemorrbage}

Hemorrhage is the most common complication of TBCB [see reference (30) for category of hemorrhage based on severity]. The overall rate of hemorrhage is $76.0 \%$, most of which are mild $(53.2 \%)$, while the rate of moderate and severe hemorrhage is $17.7-22.3 \%$ and $0.5-6 \%$, respectively $(3,5,6)$.

Hemorrhage during TBCB with endotracheal intubation is generally insignificant and require no other hemostatic measures due to the balloon catheter in place. In the rare cases of persistent bleeding, inflate the balloon and inject hemostatics through the catheter. If the balloon is displaced, redirect the balloon and handle the hemorrhage with the following measures, and these measures also apply for bleeding control in case of cryobiopsy with rigid bronchoscopy:

(I) Keep suctioning tenderly, which is usually effective to control bleeding.

(II) For hemorrhage that is heavy or cannot be controlled with suctioning, inject $1 \mathrm{~mL}$ of cold saline or 1:10,000 adrenaline, which could be given once or repeatedly; intravenous hemocoagulase and pituitrin could also be administered. However, hemocoagulase or thombin should be utilized with caution in extremely heavy bleeding to avoid formation of blood clot in the airway which may be difficult to remove and therefore result in asphyxia.

(III) For the rare cases when suctioning and hemostatic 
drugs cannot control the hemorrhage, place a balloon catheter through the flexible bronchoscope as described earlier. Another option is to place hemostatic gauze into the bronchus, but the gauze should be handled with care because displacement of it could lead to asphyxia.

(IV) Although bronchial artery embolization and surgery is rarely required for hemorrhage control, but these measures should always be prepared as a backup.

\section{Pneumothorax and mediastinal and subcutaneous emphysema}

The rate of pneumothorax and mediastinal and subcutaneous emphysema in TBCB is around 10\% (31). After each biopsy, the neck and thorax should be examined for subcutaneous crepitus and comparative percussion and auscultation of both lungs should be performed. If crepitus is present or if breath sound lowers in the biopsied side, the procedure should be terminated immediately and X-ray or ultrasound should be performed to evaluate pneumothorax and mediastinal and subcutaneous emphysema, or diagnostic thoracentesis should be carried out (15).

Mild pneumothorax and mediastinal and subcutaneous emphysema without significant shortness of breath usually resolves spontaneously with oxygen therapy and do not require specific treatment. Thoracentesis or closed pleural drainage is indicated for patients with $>30 \%$ collapse of the lung, with shortness of breath or with worsening pneumothorax, and incision and drainage at the suprasternal notch is indicated when mediastinal and subcutaneous emphysema is accompanied with shortness of breath.

\section{Infection}

Post-operative observation on patients' temperature and respiratory symptoms is important. Post-operative transient fever in general resolves spontaneously. When fever persists for over 24 hours with worsening respiratory symptoms or increased white blood cell count, pathogenic examination and antibiotics are indicated, especially in patients with extensive lesions of the lungs and with high risk of infection.

\section{Acute exacerbation of the disease}

There are few reports of acute exacerbation after TBCB
$(3,27)$. Although the connection is not yet determinate, but close observation and timely management of all patients is crucial, especially for serious cases with extensive lesions, and indications and contraindications of TBCB should be weighed carefully.

\section{Acknowledgments}

Funding: Major Science and Technology Project of the 13th Five-Year Plan (2018ZX10302302003); Specific fund for science and technology innovation on social undertakings and livelihood security by Chongqing Science and Technology Bureau (cstc2017shmsA130044); First batch of high-end workshop project for young and middle-aged talent by Chongqing Municipal Health Commission (No. 2018-2).

\section{Footnote}

Conflicts of Interest: The authors have no conflicts of interest to declare.

Ethical Statement: The authors are accountable for all aspects of the work in ensuring that questions related to the accuracy or integrity of any part of the work are appropriately investigated and resolved.

\section{References}

1. Babiak A, Hetzel J, Krishna G, et al. Transbronchial cryobiopsy: a new tool for lung biopsies. Respiration 2009;78:203-8.

2. Poletti V, Ravaglia C, Gurioli C, et al. Invasive diagnostic techniques in idiopathic interstitial pneumonias.

Respirology 2016;21:44-50.

3. Li YS, Guo SL, Yi XH, et al. Efficacy and safety of transbronchial cryobiopsy in the etiologic diagnosis of diffuse lung disease. Zhonghua Yi Xue Za Zhi 2017;97:3617-23.

4. Ussavarungsi K, Kern RM, Roden AC, et al. Transbronchial Cryobiopsy in Diffuse Parenchymal Lung Disease: Retrospective Analysis of 74 Cases. Chest 2017;151:400-8.

5. Ganganah O, Guo SL, Chiniah M, et al. Efficacy and safety of cryobiopsy versus forceps biopsy for interstitial lung diseases and lung tumours: A systematic review and meta-analysis. Respirology 2016;21:834-41. 
6. Sharp C, McCabe M, Adamali H, et al. Use of transbronchial cryobiopsy in the diagnosis of interstitial lung disease-a systematic review and cost analysis. QJM 2017;110:207-14.

7. Schuhmann M, Bostanci K, Bugalho A, et al. Endobronchial ultrasound-guided cryobiopsies in peripheral pulmonary lesions: a feasibility study. Eur Respir J 2014;43:233-9.

8. Yarmus L, Akulian J, Gilbert C, et al. Cryoprobe Transbronchial Lung Biopsy in Patients After Lung Transplantation: A Pilot Safety Study. Chest 2013;143:621-6.

9. Roden AC, Kern RM, Aubry MC, et al. Transbronchial Cryobiopsies in the Evaluation of Lung Allografts: Do the Benefits Outweigh the Risks? Arch Pathol Lab Med 2016;140:303-11.

10. Hetzel J, Maldonado F, Ravaglia C, et al. Transbronchial Cryobiopsies for the Diagnosis of Diffuse Parenchymal Lung Diseases: Expert Statement from the Cryobiopsy Working Group on Safety and Utility and a Call for Standardization of the Procedure. Respiration 2018;95:188-200.

11. Sriprasart T, Aragaki A, Baughman R, et al. A Single US Center Experience of Transbronchial Lung Cryobiopsy for Diagnosing Interstitial Lung Disease With a 2-Scope Technique. J Bronchology Interv Pulmonol 2017;24:131-5.

12. DiBardino DM, Haas AR, Lanfranco AR, et al. High Complication Rate after Introduction of Transbronchial Cryobiopsy into Clinical Practice at an Academic Medical Center. Ann Am Thorac Soc 2017;14:851-7.

13. Echevarria-Uraga JJ, Pérez-Izquierdo J, García-Garai $\mathrm{N}$, et al. Usefulness of an angioplasty balloon as selective bronchial blockade device after transbronchial cryobiopsy. Respirology 2016;21:1094-9.

14. Bango-Álvarez A, Ariza-Prota M, Torres-Rivas H, et al. Transbronchial cryobiopsy in interstitial lung disease: experience in 106 cases - how to do it. ERJ Open Res 2017. doi: 10.1183/23120541.00148-2016.

15. Jiang JY, Guo SL, Li YS. Advance in transbronchial cryobiopsy. Zhonghua Jie He He Hu Xi Za Zhi 2017;40:619-22.

16. Ravaglia C, Wells AU, Tomassetti S, et al. Transbronchial Lung Cryobiopsy in Diffuse Parenchymal Lung Disease: Comparison between Biopsy from 1 Segment and Biopsy from 2 Segments - Diagnostic Yield and Complications. Respiration 2017;93:285-92.

17. Casoni GL, Tomassetti S, Cavazza A, et al. Transbronchial Lung Cryobiopsy in the Diagnosis of Fibrotic Interstitial
Lung Diseases. PLoS One 2014;9:e86716.

18. Kropski JA, Pritchett JM, Mason WR, et al. Bronchoscopic cryobiopsy for the diagnosis of diffuse parenchymal lung disease. PloS One 2013;8:e78674.

19. Fruchter O, Fridel L, Rosengarten D, et al. Transbronchial cryobiopsy in immunocompromised patients with pulmonary infiltrates: a pilot study. Lung 2013;191:619-24.

20. Franke KJ, Linzenbold W, Nuessle D, et al. A New Tool for Transbronchial Cryobiopsies in the Lung: An Experimental Feasibility ex vivo Study. Respiration 2016;91:228-34.

21. Yarmus LB, Semaan RW, Arias SA, et al. A Randomized Controlled Trial of a Novel Sheath Cryoprobe for Bronchoscopic Lung Biopsy in a Porcine Model. Chest 2016;150:329-36.

22. Ing M, Oliver RA, Oliver BGG, et al. Evaluation of Transbronchial Lung Cryobiopsy Size and Freezing Time: A Prognostic Animal Study. Respiration 2016;92:34-9.

23. Colby TV, Tomassetti S, Cavazza A, et al. Transbronchial Cryobiopsy in Diffuse Lung Disease: Update for the Pathologist. Arch Pathol Lab Med 2017;141:891-900.

24. Ravaioli S, Bravaccini S, Tumedei MM, et al. Easily detectable cytomorphological features to evaluate during ROSE for rapid lung cancer diagnosis: from cytology to histology. Oncotarget 2017;8:11199-205.

25. Mallya V, Kumar SP, Meganathan P, et al. The utility of ROSE (rapid on-site evaluation) in endobronchial ultrasound (EBUS)-guided transbronchial needle aspiration (TBNA): Is the picture rosy? J Cytol 2015;32:230-3.

26. Viglietta L, Inchingolo R, Pavano C, et al. Ultrasonography for the Diagnosis of Pneumothorax after Transbronchial Lung Cryobiopsy in Diffuse Parenchymal Lung Diseases. Respiration 2017;94:232-6.

27. Ravaglia C, Bonifazi M, Wells AU, et al. Safety and Diagnostic Yield of Transbronchial Lung Cryobiopsy in Diffuse Parenchymal Lung Diseases: A Comparative Study versus Video-Assisted Thoracoscopic Lung Biopsy and a Systematic Review of the Literature. Respiration 2016;91:215-27.

28. Pajares V, Puzo C, Castillo D, et al. Diagnostic yield of transbronchial cryobiopsy in interstitial lung disease: a randomized trial. Respirology 2014;19:900-6.

29. Chen XB, Luo Q, Chen Y, et al. The efficacy and safety of transbronchial lung cryobiopsy in interstitial lung disease: a prospective study. Zhonghua Jie He He Hu Xi Za Zhi 2018;41:468-72. 
30. Du Rand IA, Blaikley J, Booton R, et al. British Thoracic Society guideline for diagnostic flexible bronchoscopy in adults: accredited by NICE. Thorax 2013;68 Suppl 1:i1-44.

31. Iftikhar IH, Alghothani L, Sardi A, et al. Transbronchial

Cite this article as: Guo S, Li Q, Jiang J, Luo F, Li Y, Jin F, Liu X, Wang H, Chen P, Bai C, Dai H, Huang H, Ye X, Yi X, Zhang J, Wang C, Ke M, Sun J, Feng J, Zhou H, Wu Y, Wang Z, Ma Y, Li J, Lv L, Xie B, Hohenforst-Schmidt W, Ding W, Wang X, Yang J, Cai Q, Sun P, Luo Z, Giri M; on behalf of the Working Committee on Interventional Pulmonology, Respiratory Physicians' Branch of Chinese Medical Doctor Association, Committee of Interventional Pulmonology, Chinese Society for Tuberculosis, Chinese Medical Association, Chinese Western Association of Interventional Pulmonology. Chinese expert consensus on the standardized procedure and technique of transbronchial cryobiopsy. J Thorac Dis 2019;11(12):4909-4917. doi: 10.21037/jtd.2019.12.36
Lung Cryobiopsy and Video-assisted Thoracoscopic Lung Biopsy in the Diagnosis of Diffuse Parenchymal Lung Disease. A Meta-analysis of Diagnostic Test Accuracy. Ann Am Thorac Soc 2017;14:1197-211. 